\title{
Changing trend in the management of head neck cancers during the COVID-19 pandemic
}

\author{
Pradeep Pradhan $^{1}$ (1) $\cdot$ C. Preetam ${ }^{1} \cdot$ Pradipta Kumar Parida $^{1} \cdot$ Dillip Kumar Samal ${ }^{1}$
}

Received: 16 April 2021 / Accepted: 19 May 2021 / Published online: 13 June 2021

(c) The Author(s), under exclusive licence to Springer-Verlag GmbH Germany, part of Springer Nature 2021

\begin{abstract}
Background In the present study, we have shared our experience in managing head neck cancers, especially the oral malignancies, during the crisis of COVID 19.

Materials and methods Patients with oral cancers underwent pedicle/local flaps and free flaps reconstruction based on the availability of intensive care unit and comorbidities of the patients. The clinical outcomes were compared at the end of one week, one month, and three months after the primary surgery.

Results Pedicle/local flaps were used in 25 cases and radial/fibular free flaps were used in 8 cases for the reconstruction of soft tissue defects. Patients with pedicle flap reconstruction had better clinical outcomes, including lesser ICU stay as compared to free flaps.

Conclusion Pedicle flap can be a valid alternative to the free flap for the soft tissue reconstruction in advanced oral malignancies during the COVID pandemic period in the Indian subcontinent, especially with limited infrastructure of the hospitals.
\end{abstract}

Keywords COVID $19 \cdot$ Intensive care facility $\cdot$ Pedicle flap $\cdot$ Free flap $\cdot$ Outcomes

\section{Introduction}

Management of Head Neck Cancer is a big challenge for surgeons during the period of the COVID pandemic. Different protocols have been described in the past for the optimized management of the Head Neck Cancers [1-5]. Later could be due to the advanced stage of the tumour because of the delayed presentation during the COVID 19 era as a result of quarantine and prolonged lockdown in the Indian subcontinent. During the reopening of the hospitals after the first phase of COVID 19 infection, i.e., after the month of September 2020, there was a sudden increase in the inflow of patients to the hospitals attending the Otolaryngology and head neck surgery. Although benign lesions could have been managed without much difficulty, the management of cancers affecting the head neck was a real challenge not only because of their delayed presentation but also due to the limited space in the operation theatre and the intensive care

Pradeep Pradhan

padiapradhan@gmail.com

1 Department of ENT and Head Neck Surgery, All India Institute of Medical Sciences, Bhubaneswar, Odisha 751019, India units (ICU)[3]. As majorities of the hospital beds, including the intensive care units, were diverted to the infectious COVID unit, there was an acute crisis of the beds, especially in the ICU, for the management of routine head neck malignancies. Due to the advanced stage of presentation, the operative time was found to be significantly prolonged, which later required increases in the ICU and the hospital stay of the operated patients. Again with the increase in the hospital stay, the chance of transmission of the infection to the healthcare workers gets increased, especially the patients admitted to the ICU [6]. Hence the availability of ICU stay plays a vital role in the management of advanced head neck cancers during the COVID 19 crisis [7], which is often the determining factor for the soft tissue reconstruction in head neck cancers. As the free flap reconstruction after the excision of the tumour usually requires ICU stay, patients with oral cancers can be alternatively managed with pedicle/local flaps during the COVID crisis. In the present study, we have shared our experience in the management of head neck cancers during the COVID 19 pandemic period. 


\section{Materials and methods}

It is a retrospective analysis of all the head and neck cancers that were operated on just after the peak of COVID 19 infection, i.e., from September 2020 to March 2021 in the Department of Otolaryngology and head neck surgery, All India Institute of Medical Sciences Bhubaneswar. Diagnosed cases of head neck cancers were included in the study.

\section{Surgical technique}

After the informed and written consent from the patient, the surgery was performed on each patient. All the procedures were done by a team of doctors, adequately experienced in head neck and reconstructive surgeries. The surgical procedures followed a fixed protocol of safety measures to COVID 19 infection. Complete PPE (personal protective equipment), including face shield, was universally used by the healthcare personals during the surgeries. All patients with negative COVID report (Reverse transcription-polymerase chain reaction) with the nasopharyngeal swab had undergone surgery. Patients with positive swab tests for COVID were admitted to the infectious disease department until three consecutive tests become negative. Wide local excision of the lesion with and without neck dissection was performed in all the cases of head neck cancers. Soft tissue reconstruction was advised in patients with oral cancers, which were decided based on the availability of the spaces in the major operation theatre and intensive care units and the associated comorbidities of the patients. Pedicle flaps (Pectoralis Major Myocutaneous flap, Deltopectoral flap)/local flaps (nasolabial/Abbe-Estlander flap) were used for the soft tissue reconstruction due to the unavailability of the ICU bed (Figs. 1, $2,3)$. In contrast, free flap reconstruction was performed when the ICU beds were found available for the monitoring of the patient in the postoperative period. In the free tissue transfer, radial forearm flap was used for selected cases of

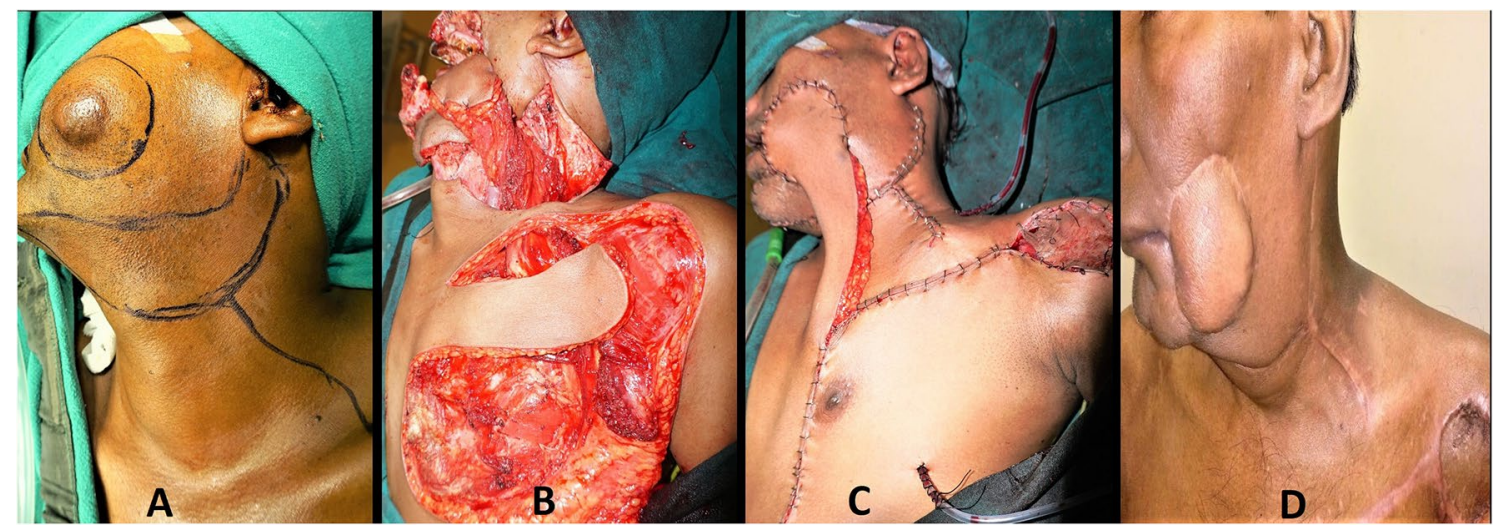

Fig. 1 a Shows carcinoma of Left buccal mucosa involving the overlying skin (stage IV), b combined used of Pectoralis major myocutaneous flap and Deltopectoral flap reconstruction, $\mathbf{c}$ closure of the surgical defect, $\mathbf{d}$ photograph of the patient 4 weeks after the chemoradiation

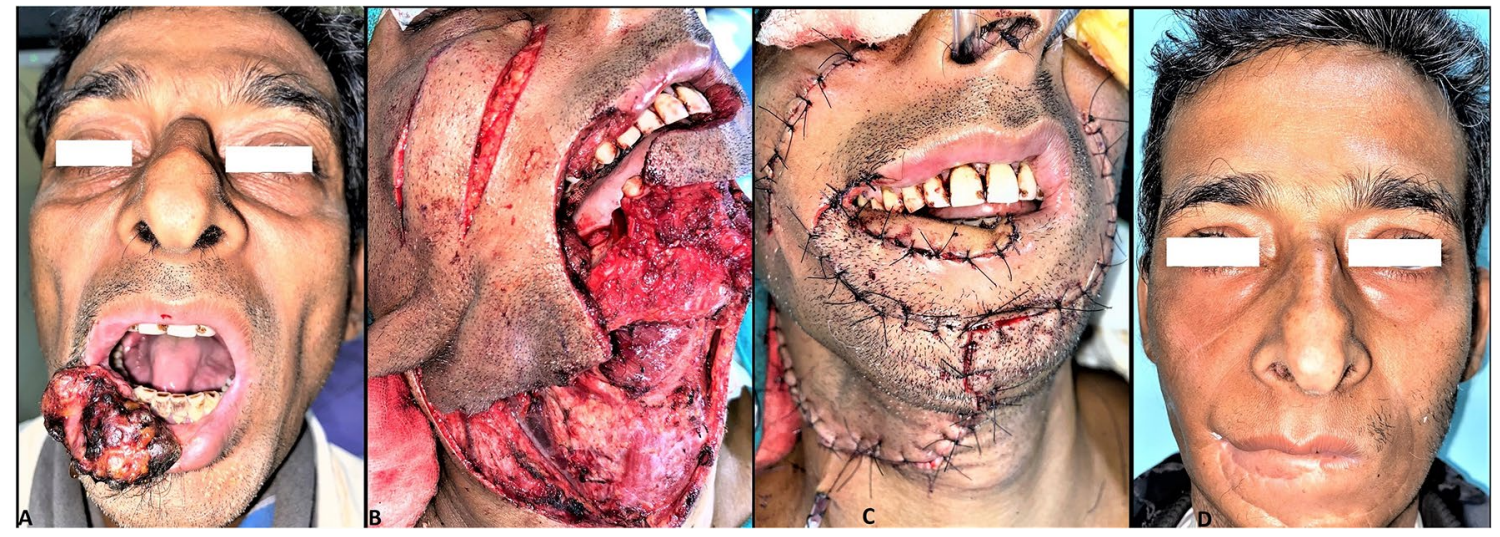

Fig. 2 a Shows carcinoma of the lower lip, b Intraoperative photo showing the soft tissue defect after excision of the tumour, $\mathbf{c}$ Elevation of the left Karapandzic flap and right Nasolabial flap, $\mathbf{d}$ photograph of the patient one month after the primary surgery 


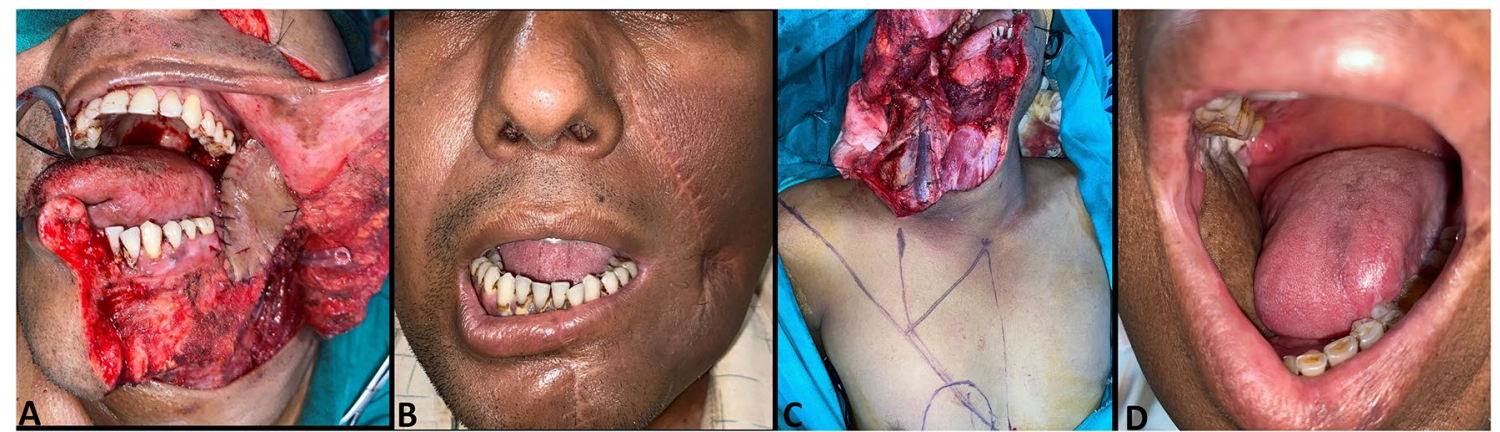

Fig. 3 a Shows reconstruction with nasolabial flap for a malignancy involving left retromolar trigone, b Photograph of the patient one month after the radiotherapy. c Reconstruction of Pectoralis major

carcinoma of the tongue, buccal mucosa, and Osseo-cutaneous (free fibula) composite flap was selected cases where the mandibular bone had to be excised during the surgery. The patients were discharged after the removal of the neck drain in the postoperative period and were advised to follow up in the head neck clinic after one week, one month and three months after the surgery. In the follow-up period, each patient was assessed for the major and minor complications and the recurrence of the disease.

\section{Results}

Of 44 patients operated during the unlocking of COVID 19, 32 patients were male $(72.7 \%)$ and $12(27.3 \%)$ were female. The age of the patients ranged from 30 to 63 years (mean age $41.34 \pm 7.69$ years). The average age of the male patients was $41.47 \pm 8.33$ years and the average age of the females was $41.00 \pm 5.95$ years. The mean duration of the disease was 7 months (range 1-9 months). The demographic profile of the patients with head neck cancer has been shown in Table 1. Of the 44 patients, 30(68.18\%) were chronic smokers and 9(20.45\%) patients had a history of alcohol intake. The average Karnofsky performance status score was 60(range 50-90), Appendix (1). Out of 44 patients, $12(27.27 \%)$ patients were found to be affected with diabetes mellitus, 7(15.90\%) patients with chronic lung disease and one patient had a history of pulmonary tuberculosis during their initial presentation to the outpatient department of head neck surgery. Of the 44 patients, 33(75\%) patients were diagnosed with carcinoma of oral $06(13.63 \%)$ cases were carcinoma of the larynx, $5(11.36 \%)$ cases were diagnosed as carcinoma of the maxillary sinus. The frequencies of malignancies in various subsites in the head and neck region have been demonstrated in Fig. 4. The average duration of the disease before attending the hospital was 07 months (range 01-09 months). Of 44 patients with head neck cancers, 29(65.90\%) patients presented with stage IV
Myocutaneous flap to repair the mandibular defect (Right) in a case of carcinoma of the oral cavity. d Photograph of the oral cavity of the patient one month after the radiotherapy

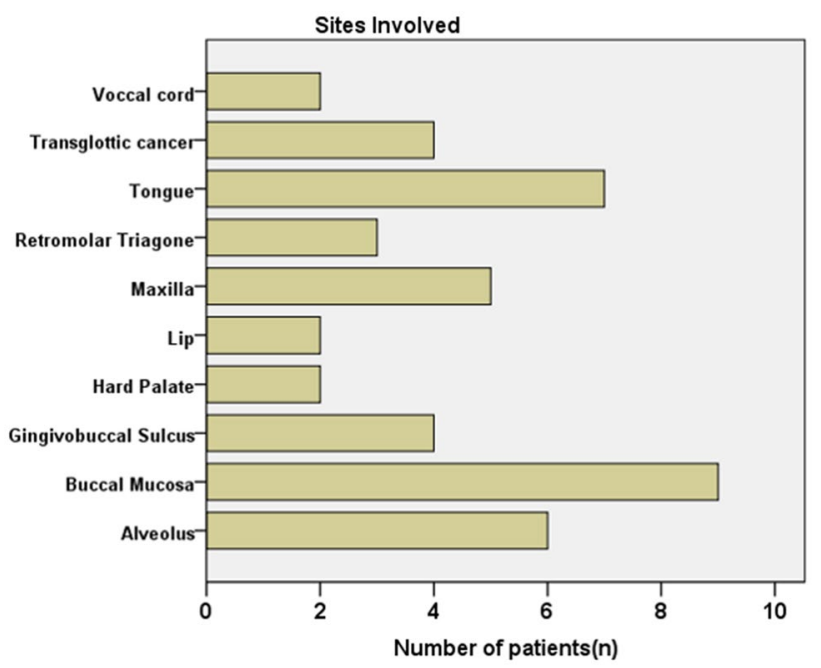

Fig. 4 The frequencies of malignancies in different subsites by cancers in the head and neck region

disease, and 13(29.54\%) patients as stage III and 2(4.54\%) patients with stage I disease after the confirmation in the final histopathological examination (Fig. 5). The majority of the cancers $(65.90 \%)$ affecting the oral cavity were diagnosed with stage IV disease. The differential staging of the head neck tumours with respect to the various subsites has been demonstrated in Table 2. Pedicle flap (Pectoralis major myocutaneous flap with and without Deltopectoral flap) was used in $19(43.2 \%)$ cases and $8(18.2 \%)$ cases required free flap (radial/fibular free flap) for the reconstruction of the surgical defect. A total of $6(13.6 \%)$ patients were managed with local flaps (Abbe-Estlander flap/Nasolabial flap) and $11(25 \%)$ patients did not require any flap for the reconstruction of the surgical defect. Amongst them, 5 patients were presented with sinonasal malignancies and 6 patients were presented with laryngeal cancers. The mean surgical time of all the head neck cancers irrespective of reconstruction was detected to be $328.64 \pm 122.18 \mathrm{~h}$ (range $45-600 \mathrm{~min}$ ). The 


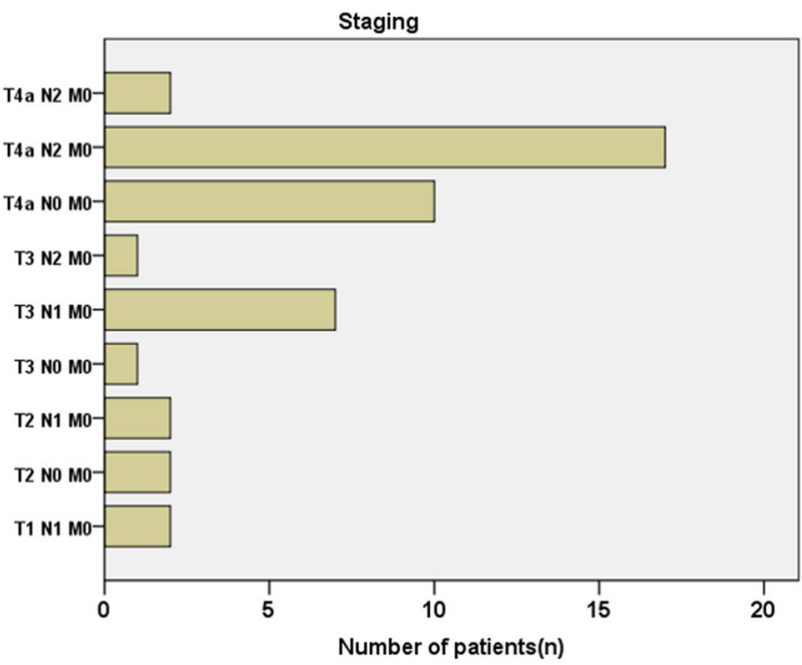

Fig. 5 TNM Staging of tumours affecting head and neck during the COVID 19 pandemic era

Table 1 Demographic data of head neck malignancies during the COVID pandemic period $[n=44]$

\begin{tabular}{lll}
\hline Characters & $\begin{array}{l}\text { Number of } \\
\text { patients }\end{array}$ & Range \\
\hline Sex & & \\
Male & 32 & \\
Female & 12 & $30-63$ \\
Mean age of patients (Year) & 41 & $01-09$ \\
Mean duration of the disease (months) & 07 & $03-05$ \\
Minimum duration of follow-up (months) & 3 & \\
Smoking & & \\
Yes & 30 \\
No & 12 & \\
Alcohol & & \\
Yes & 09 \\
No & 35 \\
Primary sites (Oral cavity) & & \\
Buccal mucosa & 09 \\
Alveolus & 06 \\
Gingivobuccal sulcus & 04 \\
Retro molar triagone & 03 \\
Tongue & 07 \\
Hard palate & 02 \\
Lower lip & 02 \\
Primary sites(Larynx) & & \\
SCC of true vocal cord & 02 & \\
Pransglottic SCC & & \\
Maximary sites (Paranasal sinus) & & \\
\hline
\end{tabular}

SCC Squamous Cell Carcinoma
Table 2 Shows the TNM staging of all of head neck cancers

\begin{tabular}{lllll}
\hline Characteristics & Primary site & \multicolumn{3}{l}{ TNM Staging (No of cases) } \\
\cline { 3 - 5 } Oral cancers & Sub sites & Stage IV & Stage III & Stage I/II \\
\hline \multirow{6}{*}{ Buccal mucosa } & 6 & 3 & 0 \\
& Alveolus & 4 & 2 & 0 \\
& GBS & 3 & 1 & 0 \\
& RMT & 2 & 1 & 0 \\
Larynx & Tongue & 4 & 3 & 0 \\
& Hard palate & 0 & 2 & 0 \\
& Lower lip & 1 & 1 & 0 \\
& Sub sites & Stage IV & Stage III & Stage I/II \\
& Vocal cord & 0 & 0 & 2 \\
& Subglottic & 4 & 0 & 0 \\
& Sub sites & Stage IV & Stage III & Stage I/II \\
& Maxillary sinus & 5 & 0 & 0 \\
\hline
\end{tabular}

$G B S$ :Gingivobuccal Sulcus, $R M T$ :Retromolar trigon

average duration of surgical procedure for oral cancer was found to be $361.36 \pm 116.22 \mathrm{~min}$ (range 180-600 $\mathrm{min}$ ). When the duration of the operative time was compared between the patients with pedicle/local flaps and free flap, the pedicle flaps reconstruction required a significantly lesser operative time as compared to the free flap $(\mathrm{p}=0.00)$. Again, one patient with the pedicle flap and all the patients with free flap reconstruction required ICU care after the primary surgery $(p=0.00)$, as shown in Table 3 . Of the 8 cases who had undergone free flap reconstruction, 2 cases required reexplanation of the surgery in the immediate postoperative period due to early ischemic change in the flap. None of the patients in the pedicle flap repair needed reexploration of the surgery in the postoperative period $(p=0.03)$. The mean duration of the hospital stay was approximately $5.84 \pm 1.72$ days (range 4-11 days), considering all the head neck cancers. Again, the mean duration of the hospital stays in patients undergoing pedicle flap/local flap and the free flap reconstruction were found to be $5.28 \pm 0.93$ and $8.12 \pm 1.88$ days, respectively $(\mathrm{p}=0.00)$. Of the 29 flaps that underwent pedicle/local flap reconstruction, one patient had partial necrosis of the skin island, two patients had wound infection, one had a hematoma in the neck within 7 days of the primary surgery. Similarly, one patient from the free flap reconstruction (free fibular flap) had complete flap rejection $48 \mathrm{hr}$ after the primary surgery (Table 4).

At the second follow-up (one month), one patient presented with orocutaneous fistula and one had salivary fistula in the free flap group, which was managed with the conservative approach. No significant complications were noticed at the third follow-up period ( 3 months) in any of the patients in either group. Neoadjuvant chemotherapy was given in 3 patients with oral cavity malignancies due to delay in the surgery. All the patients with stage IV tumours and 9 
Table 3 The difference in the clinical findings between Pedicle flaps/Local flaps vs Free flaps

\begin{tabular}{llll}
\hline & Soft tissue reconstruction & & \\
\hline Characteristics & Pedicle flaps/Local flap & Free flap & $p$ value, 95\% CI, SEM \\
Duration of surgery (minutes) & $307.80 \pm 69.25$ & $528.75 \pm 55.14$ & 0.000, CI(275.90-165.99), 26.94 \\
Duration of hospital stay(days) & $5.28 \pm 0.93$ & $8.12 \pm 1.88$ & 0.00, CI(-3.85--1.83), 0.49 \\
Requirement of ICU (No of patients) & 01 & 08 & 0.00 \\
Reexploration of surgery(No of patients) & 00 & 02 & 0.00 \\
\hline
\end{tabular}

$I C U$ : Intensive Care Unit, $C I$ : confidence interval, SEM: standard error of mean

Table 4 Complications between the pedicle flap/Local flap vs Free flap reconstruction during the follow-up periods

\begin{tabular}{lllll}
\hline Follow-up & Complications & $\begin{array}{l}\text { Pedicle flap/ } \\
\text { local flap (n) }\end{array}$ & Free flap(n) & $p$-value \\
\hline 1 week & Flap necrosis & 00 & 01 & 0.224 \\
& Wound infection & 02 & 00 & \\
& Hematoma & 01 & 00 & \\
\multirow{1}{*}{1 month } & Orocutaneous & 00 & 01 & 0.175 \\
& fistula & & & \\
& Salivary fistula & 00 & 01 & \\
\hline
\end{tabular}

patients in stage III tumour underwent adjuvant radiotherapy due to extranodal/perineural extension of the tumour in the final histopathological examination. The minimum followup period was three months (range 3-5 months). None of the patients had clinical or radiological evidence of disease recurrence till the last follow-up period.

\section{Discussion}

Cancers of the oral cavity are considered a significant public health problem worldwide, accounting for approximately 447,571 new cases and 228,389 deaths in a year [8]. Majorities of the cancer patients belong to low- and middleincome groups of the population [9]. Approximately 30\% of the global burden of oral cancer is contributed by India, where it is the most common cancer affecting the whole body in men [8]. With the emergence of the COVID 19, management of head neck cancers caused a significant public health crisis where later was declared by WHO a public health emergency in the globe. Again, cancer surgery with chemotherapy often causes immunosuppression to the patients making them susceptible to the development of severe COVID pneumonia attributing to significant mortality in the postoperative period [10-14]. Although early detection of head neck cancers can be curative, management of advanced cancer is a big challenge in COVID 19 pandemic time due to the advanced stage of the disease as a result of prolonged lockdown, causing significant mortality and morbidity to the patients. Hence during the unlocking period after the COVID 19 surge, the majority of the patients presented with stage IV tumour. Although various literature has been produced in the past documenting different guidelines for the treatment of cancer during the COVID pandemic period $[1,5,15-17]$, it is always a challenge to manage the advanced head neck malignancies, with limited infrastructure and resources in the COVID pandemic, especially in a developing country like India. Hence the guideline of cancer management is mainly customized based on the availability of the hospital resources, workforce and infrastructure, especially in the COVID crisis. Although neoadjuvant chemotherapy is not routinely recommended in head neck malignancies, it can be an optional interim treatment option to halt the progress of the disease, especially in stage IV oral and oropharyngeal cancers.

As noticed in the current series, of 33 cases of oral cancers, 5 patients were detected as COVD positive with a swab test. Cisplatin-based chemotherapy (at least two cycles) was given in 3 cases till their COVID report becomes negative. Although the standard surgical treatment offered to the patients of head neck malignancies should be pretty similar to the non-COVID period, the management protocol we adopted was quite different with regards to the soft tissue reconstruction in the advanced stage of the disease. Of 33 patients with oral malignancies, 25 had undergone soft tissue reconstruction with pedicle/local flaps as the primary mode of reconstruction either due to the lack of ICU beds or associated with chronic comorbidities where the free flap reconstruction was not performed. As observed in the study, free flap reconstruction not only creates a significant burden to the ICU space, it exposes both the patients and the healthcare professional for the COVID 19 infection [18]. Although free flaps are thought to be a better alternative to pedicle flaps even in advanced oral malignancies, their utility is critically limited, especially in elderly patients with significant comorbidities, as observed in our study. All 25 cases have undergone single-stage excision of the tumour and soft tissue reconstruction either with the pedicle flap (Pectoralis Major Myocutaneous flap, Deltopectoral flap) or local flaps (Abbe-Estlander flap/Nasolabial flap), especially in cases where free flap reconstruction was not feasible. Again, 11 patients, including carcinoma of the larynx and 
sinonasal cavity, had undergone radical surgery, i.e., total laryngectomy and maxillectomy, respectively. When the surgical time was considered, reconstruction of the pedicle flap was quicker in contrast to the free flap and later had better survival compared to the free flap. As demonstrated in the present study, the surgical time was significantly reduced in the pedicle/local flap reconstruction $(\mathrm{p}=0.00)$. In the current case series, almost all the pedicle flaps were successfully repaired without any rejection of the flap compared to the free flaps where one had complete necrosis 48 after the primary surgery. There was no significant difference in complications between the two groups of patients in the postoperative period $(p \geq 0.05)$. Again, each of the patients with free flaps required ICU backup compared to the pedicle flaps, where only one patient was shifted for ICU for short-term monitoring of the vitals $(p=0.00)$. During the COVID 19 pandemic, creating a space in the ICU was a big challenge because of the diversion of the majority of the ICU beds towards critically ill patients with severe COVID pneumonia. Again, with the existing infrastructure and workforce, it was really a difficult task to expand the intensive care unit, especially in the Indian subcontinent, where the requirement of the ICU stay was significantly increased with the increase of the COVID 19 active cases [19]. Depending upon the basic infrastructure and comorbidities of the patients, majorities of the advanced cancer cases were successfully managed by pedicle flap reconstruction to overcome the huge patient load coming from a low socioeconomic background where intensive care monitoring is a major limiting factor for soft tissue reconstruction. Again, a longer hospital stay of the patients carries a significant risk of COVID infection, and shorter hospital stays might reduce the transmission of the disease among the health care workers [20,21]. As demonstrated in the present study, a significant reduction of the ICU stay was noted in patients with pedicle flap reconstruction as compared to the free flap repair $(p=0.00)$. Due to the high risk of infection and limited ICU beds, majorities of the patients often get postponed even with the advanced stage of treatment. In contrast, in the present study, none of the patients was referred or postponed even with the limited capacity of the ICU bed, which could have been possible due to the alternative soft tissue reconstruction (pedicle flap/ local flaps) even with advanced stage of the disease. As demonstrated in the present case series, none of the health care workers had COVID infection during the course of the treatment, which might be due to the shorter hospital stay, and that could have been possible due to the use of the pedicle flap/local flap for the soft tissue reconstruction.

With respect to flap survival, the flap uptake rate was almost $100 \%$ in the pedicle flap patients. Only one patient had skin necrosis, 2 patients had wound dehiscent and 1 patient developed a hematoma in the neck after $48 \mathrm{~h}$ of surgery which was managed with the conservative approach as supported by similar studies [22-24]. Various recommendations have been proposed in the past for the management of head neck malignancies [25], and no definitive policy is still established for the soft tissue reconstruction during the COVID-19 pandemic time. Hence the guideline for the management of head neck cancer can be modified depending on the stage of the tumour, the comorbidities, availability of resources, including the number of ICU space and hospital stay as demonstrated in our study. It is evident in our case study that the COVID 19 infection significantly affected the surgical practice, especially for head neck cancers; the change in the trend needs to be carefully monitored, which may be adopted during the second wave of the COVID crisis. The strategic shift in the reconstructive procedures has allowed us to save the precious resource without jeopardizing the validity and the functional results, and the viewpoint can be considered during the COVID outbreak in the future. In spite of the small sample size and short follow-up period, we have highlighted the optimized management of head neck cancer with the availability of resources, including the ICU spaces in the Indian subcontinent, where most of the patients come from a low socioeconomic background.

\section{Conclusion}

Management of Head Neck Cancer is a big challenge for surgeons during the period of the COVID pandemic. Patients usually present at an advanced stage of the tumour due to the delayed presentation during the COVID 19 era as a result of quarantine and prolonged lockdown in the Indian subcontinent. The guideline for the management of head neck cancer can be modified depending on the stage of the tumour, the comorbidities, availability of resources, including the number of ICU space and hospital stay as demonstrated in our study.

Although the free flap reconstruction often requires ICU stay, patients with oral cancers can be alternatively managed with pedicle /local flaps enabling comparable clinical outcomes during the pandemic of COVID 19 with decreasing the hospital stay. The strategic change in the reconstructive procedures has allowed us to save the precious resource without jeopardizing the validity and the functional results in the management of head neck cancers, and the viewpoint can be considered during the COVID outbreak in the future. Although it is a small sample size with a short follow-up period, here we have highlighted the optimized management of head neck cancer with the limited availability of resources, including the ICU spaces in the Indian subcontinent, where most of the patients come from a low socioeconomic background. 


\section{Appendix 1: Karnofsky performance status score.}

The Karnofsky score measures patient performance of activities of daily living

Karnofsky scores at the time of tumour diagnosis have the best survival and quality of life over the course of their illness

100- Normal, no evidence of disease

90- Able to perform normal activity with only minor symptoms

80- Normal activity with effort, some symptoms

70- Able to care for self but unable to do normal activities

60- Requires occasional assistance, cares for most needs

50- Requires considerable assistance

40- Disabled, requires special assistance

30- Severely disabled

20- Very sick, requires active supportive treatment

10- Moribund

Acknowledgements The authors would like to acknowledge that the study was conducted in All India Institute of Medical Sciences, Bhubaneswar, in the Department of ENT and Head Neck Surgery. There are no conflicts of interest among the authors. This research has not been supported by any financial agency or funding organization.

Funding The study was not funded by any organization or institution.

\section{References}

1. Shaw R. COVID-SURG cancer. British Association of Head \& Neck Oncologists (BAHNO). https://www.bahno.org.uk/ covidsurg cancer.aspx. Accessed 11 June 2020

2. COVIDSurg Collaborative. Mortality and pulmonary complications in patients undergoing surgery with perioperative SARSCoV-2 infection: an international cohort study. Lancet 2020. https://doi.org/10.1016/S0140-6736(20)31182-X. Accessed 11 June 2020

3. Day AT, Sher DJ, Lee RC et al (2020) Head and neck oncology during the COVID-19 pandemic: reconsidering traditional treatment paradigms in light of new surgical and other multilevel risks. Oral Oncol 105:104684

4. Fakhry N, Schultz P, Morinière S et al (2020) French consensus on management of head and neck cancer surgery during COVID-19 pandemic. Eur Ann Otorhinolaryngol Head Neck Dis 137:159-160

5. OMFS and COVID-19. British Association of Oral and Maxillofacial Surgeons (BAOMS). https://www.baoms.org.uk/profession als/omfs and covid-19.aspx. Accessed 11 June 2020.

6. Zhao Z, Gao D (2020) Precaution of 2019 novel coronavirus infection in department of oral and maxillofacial surgery. Br J Oral Maxillofac Surg 58:250-253

7. Montero PH, Patel SG (2015) Cancer of the oral cavity. Surg Oncol Clin N Am 24:491-508

8. Warnakulasuriya S, Greenspan JS. (2020) Epidemiology of oral and oropharyngeal cancers. In: WarnakulasuriyaGreenspaneditors Textbook of oral cancer. Berlin: Springer. p. 5-22.
9. Nagao T, Warnakulasuriya S (2020) Screening for oral cancer: Future prospects, research and policy development for Asia. Oral Oncol 105:104632. https://doi.org/10.1016/j.oraloncology. 2020.104632

10. Montopoli M, Zumerle S, Vettor R, Rugge M, Zorzi M, Catapano CV, Carbone GM, Cavalli A, Pagano F, Ragazzi E, PrayerGaletti T, Alimonti A (2020) Androgen-deprivation therapies for prostate cancer and risk of infection by SARS-CoV-2: a population-based study ( $\mathrm{N}=4532)$. Ann Oncol 31(8):1040-1045

11. Au L, Boos LA, Swerdlow A, Byrne F, Shepherd STC, Fendler A, Turajlic S (2020) CAPTURE investigators. Cancer, COVID-19, and Antiviral Immunity: The CAPTURE Study. Cell. 183(1):4-10. https://doi.org/10.1016/j.cell.2020.09.005

12. Venkatesulu BP, Lester S, Hsieh CE, Verma V, Sharon E, Ahmed M, Krishnan S (2020) Low-dose radiation therapy for COVID-19: promises and pitfalls. JNCI Cancer Spectr 5(1):103. https://doi.org/10.1093/jncics/pkaa103

13. Dhawan G, Kapoor R, Dhawan R, Singh R, Monga B, Giordano J, Calabrese EJ (2020) Low dose radiation therapy as a potential life saving treatment for COVID-19-induced acute respiratory distress syndrome (ARDS). Radiother Oncol 147:212-216

14. Lara PC, Burgos J, Macias D (2020) Low dose lung radiotherapy for COVID-19 pneumonia. The rationale for a costeffective anti-inflammatory treatment. Clin Transl Radiat Oncol 23:27-29. https://doi.org/10.1016/j.ctro.2020.04.006

15. COVIDSurg Collaborative (2020) Mortality and pulmonary complications in patients undergoing surgery with perioperative SARS-CoV-2 infection: an international cohort study. Lancet 396(10243):27-38

16. Day AT, Sher DJ, Lee RC, Truelson JM, Myers LL, Sumer BD, Stankova L, Tillman BN, Hughes RS, Khan SA, Gordin EA (2020) Head and neck oncology during the COVID-19 pandemic: Reconsidering traditional treatment paradigms in light of new surgical and other multilevel risks. Oral Oncol 105:104684. https://doi.org/10.1016/j.oraloncology.2020.104684

17. Fakhry N, Schultz P, Morinière S, Breuskin I, Bozec A, Vergez S, de Garbory L, Hartl D, Temam S, Lescanne E, Couloigner V, Barry B; French Society of Otorhinolaryngology, Head and Neck Surgery (SFORL); French Society of Head and Neck Carcinology (SFCCF). French consensus on management of head and neck cancer surgery during COVID-19 pandemic. Eur Ann Otorhinolaryngol Head Neck Dis. 2020;137(3):159-160.

18. Zou L, Ruan F, Huang M, Liang L, Huang H, Hong Z, Yu J, Kang M, Song Y, Xia J, Guo Q, Song T, He J, Yen HL, Peiris M, Wu J (2020) SARS-CoV-2 viral load in upper respiratory specimens of infected patients. N Engl J Med 382(12):1177-1179

19. Wu Z, McGoogan JM (2020) Characteristics of and important lessons from the coronavirus disease 2019 (COVID-19) outbreak in China: summary of a report of 72314 cases from the chinese center for disease control and prevention. JAMA 323(13):1239-1242

20. Day M (2020) Covid-19: Italy confirms 11 deaths as cases spread from north. BMJ 26(368):m757. https://doi.org/10.1136/ bmj.m757

21. Tuite AR, Ng V, Rees E, Fisman D (2020) Estimation of COVID-19 outbreak size in Italy. Lancet Infect Dis 20(5):537

22. Colman MF, Zemplenyi J (1986) Design of incisions for pectoralis myocutaneous flaps in women. Laryngoscope 96(6):695-696

23. Brusati R, Collini M, Bozzetti A, Chiapasco M, Galioto S (1988) The pectoralis major myocutaneous flap. Experience in 100 consecutive cases. J Craniomaxillofac Surg 16(1):35-9

24. Pradhan P, Samal S, Preetam C, Samal DK, Parida PK (2018) Pectoralis major myocutaneous flap reconstruction for the mandibular defects in advanced oral cavity malignancies: a retrospective study of 30 cases. Indian J Otolaryngol Head Neck Surg 70(3):415-420 
25. Givi B, Schiff BA, Chinn SB, Clayburgh D, Iyer NG, Jalisi S, Moore MG, Nathan CA, Orloff LA, O'Neill JP, Parker N, Zender C, Morris LGT, Davies L (2020) Safety recommendations for evaluation and surgery of the head and neck during the COVID-19 pandemic. JAMA Otolaryngol Head Neck Surg 146(6):579-584
Publisher's Note Springer Nature remains neutral with regard to jurisdictional claims in published maps and institutional affiliations. 